\title{
LA EDUCACIÓN ARGENTINA EN ÉPOCAS DE LA ÚLTIMA DICTADURA MILITAR: REGIONALIZACIÓN Y DESCENTRALIZACIÓN DEL NIVEL PRIMARIO DE EDUCACIÓN (1976-1983)
}

\author{
Romina de Luca \\ Universidad de Buenos Aires (Argentina)
}

\begin{abstract}
RESUMEN: En el presente artículo analizamos una serie de transformaciones educativas implementadas en Argentina durante la última dictadura militar (1976-1983). Específicamente nos ocupamos del proceso de regionalización curricular en la llamada educación básica obligatoria o primaria promovido para todo el país desde el Ministerio de Educación nacional. Si bien lo actuado en esos años encuentra antecedentes previos intentaremos ver aquí por qué dicho proceso se consolidó en esos años sintetizando experiencias anteriores. En segundo lugar, intentaremos mostrar la confluencia entre el proceso de cambio curricular y lo actuado en materia de gestión y administración educativa; ambos procesos en relación a mejorar la satisfacción de las demandas productivas locales de fuerza de trabajo. Nuestro corpus documental lo conformaron documentos oficiales publicados por el Ministerio de Educación de la Nación argentina, en particular, las actas de las Asambleas del Consejo Federal de Educación; documentos elaborados por distintos organismos nacionales y programas de asistencia técnica, Circulares y Resoluciones internas, discursos y declaraciones formulados por protagonistas clave, datos estadísticos, legislación y prensa periódica de tirada nacional.
\end{abstract}

PALABRAS CLAVE: Historia de la educación, regionalización curricular, reformas, estrategia, síntesis.

\section{EDUCATION IN ARGENTINA TIMES LAST MILITARY DICTATORSHIP: REGIONALIZATION AND DECENTRALIZATION PRIMARY LEVEL OF EDUCATION (1976-1983)}

\footnotetext{
ABSTRACT: In this paper we analyze a series of educational changes implemented in Argentina during the military dictatorship (1976-1983) by the national government. In particular we address the regionalization
} 
process called curriculum in primary and compulsory basic education for all the country promoted by the Ministry of Education. Secondly, we will attempt to show the convergence of the process of curriculum change and acted in management and educational administration, both processes in relation to better meet the demands of local productive workforce. Our corpus of documents was made up of official documents issued by the Ministry of Education of the Nation Argentina, in particular, records of the Assemblies of the Federal Council of Education; documents prepared by various national and technical assistance programs, Circulars and internal, speeches and statements made by key players, statistics, legislation and periodical press of national circulation.

KEYWORDS: History of education, curricular regionalization, reforms, strategies, synthesis.

Recibido: 07/06/2012

Aceptado: 22/10/2012

\section{INTRODUCCIÓN}

En el presente artículo analizamos una serie de transformaciones educativas implementadas en Argentina durante la última dictadura militar. El período bajo estudio comprende la etapa iniciada el 24 de marzo de 1976, con la instauración del poder militar en dicho país, hasta la apertura del proceso electoral y el recambio de régimen hacia 1983. Dentro de la gran serie de cambios encarados en materia educativa en esos años, aquí nos abocamos a uno de crucial importancia: el proceso de regionalización curricular en la llamada educación básica'. De este modo, nuestro observable lo constituye el nivel primario de educación obligatoria. El objetivo principal del artículo consiste en reconstruir los procesos de reforma encarados por el Estado nacional en materia de organización curricular. En segundo término, observar si existe algún grado de interrelación con los cambios simultáneos que se desarrollaron en la administración del nivel, a partir de la transferencia de la gestión y el control de los establecimientos educativos nacionales a las provincias, proceso conocido como "descentralización".

A modo de hipótesis, sostenemos que lo actuado en esos años no constituyó una novedad sino, más bien, un momento de síntesis de diversos ensayos pedagógicos implementados previamente en dicho país. Por ello, afirmamos que la última dictadura militar recogió experiencias anteriores que, hasta ese momento, venían desa-

1. Existe un profuso debate internacional en torno al concepto de regionalización y sus diferencias con la llamada "nuclearización" y con la "descentralización". En el presente artículo definimos "regionalización" como la acción ejercida por el Estado nacional sobre el currículum de enseñanza obligatorio proceso a partir del cual se prescriben nociones mínimas de conocimientos obligatorios comunes para todo el país fijadas por el Estado central (en este caso para el nivel primario de educación) para que, en segundo término, esas nociones sean tomadas como indicadores de contenidos a ser desarrollados luego de ser redefinidos por cada región o localidad del país según sus propias necesidades de enseñanza. 
rrollándose de modo experimental. Su intervención zanjó un estado de discusión y de debate previo generalizándose una propuesta para el conjunto del país. Cabe destacar que, la marca sobre el sistema educativo de la última dictadura militar perduró aún depuesto el régimen castrense. Por lo tanto, su legado excede al personal político de turno. Retomaremos este punto hacia las conclusiones.

Para la reconstrucción del período nos basamos en fuentes tales como documentos oficiales publicados por el Ministerio de Educación de la Nación argentina, en particular, las actas de las Asambleas del Consejo Federal de Educación. También nos servimos de documentos elaborados por distintos organismos nacionales y programas de asistencia técnica, Circulares y Resoluciones internas, discursos y declaraciones formulados por protagonistas clave, datos estadísticos, legislación y prensa periódica de tirada nacional.

Consideramos que este momento histórico reviste interés por varios motivos. En primer lugar, la regionalización curricular, encarada a nivel nacional, no ha sido revisada profundamente por la historiografía educativa argentina en la etapa aquí elegida. Los estudios sobre educación y dictadura en el país resultan más bien recientes, primando o bien miradas muy generales o bien estudios de caso más específicos. Una excepción a tal situación se encuentra en el estudio de Laura Rodríguez para el caso de la provincia de Buenos Aires. La autora se ocupa de analizar las transformaciones del currículum en esa jurisdicción trazando algunos puntos de continuidad con proyectos anteriores (Rodríguez, 2008). En segundo lugar, el proceso confluyó con una serie de modificaciones en la administración del sistema educativo que sí fueron objeto de análisis más o menos abundante (Borthwick de Maltoni, 1995; Bravo, 1994; De Luca, 2008; Feldfeber e Ivanier, 2003; Paviglianiti, 1988; Salonia, 1996). Sin embargo, la historiografía no se ha interrogado aún si la descentralización administrativa tuvo correlato en otros aspectos de la vida escolar tales como la organización del currículum. Intentaremos mostrar aquí la correspondencia entre ambos fenómenos. En tercer término, este momento de la historia permite iluminar la serie de mutaciones producidas en el sistema educativo argentino en los años noventa -momento principalmente determinado por la sanción de la Ley Federal, en lo que refiere a educación básica, y a la culminación de los procesos de transferencia de establecimientos educativos nacionales a órbita provincial de los niveles medio y superior-. En lo que refiere a la Ley Federal, se reconstruye sus puntos de contacto con la reforma española perdiéndose de vista la extensa prehistoria de esos cambios, situación que limita la construcción de una explicación global que intente dar cuenta de las tendencias profundas que afectan al sistema.

\section{EL PROCESO DE REORGANIZACIÓN NACIONAL EN LA HISTORIA ARGENTINA Y EL ROL ESPECÍFICO RESERVADO A LA EDUCACIÓN}

A los efectos de entender el rol de la educación en el período estudiado debemos, en primer término, señalar los rasgos más salientes de esa etapa. El golpe de marzo de 1976 fue protagonizado por los sectores más concentrados de la burguesía argentina, tanto financiera, industrial como agropecuaria. Ellos se pusieron a la cabeza de una alianza política -nosotros la denominaremos aquí "alianza militar"que logró acaudillar, detrás de sí, al resto de las fracciones de ésa clase y a otras pro- 
venientes de la pequeña burguesía. Su principal objetivo era el aniquilar a la fuerza social revolucionaria que se había conformado entre 1969 y el golpe para cerrar la brecha abierta tras el Cordobazo ${ }^{2}$. El proceso remite, necesariamente, a la crisis hegemónica y de acumulación de capital que en la Argentina se expresó luego del derrocamiento del segundo gobierno de Juan Domingo Perón, en septiembre de 1955. Pero el golpe de 1976 resulta cualitativamente diferente. En tanto, cerrar la crisis implicó el aniquilamiento físico -producir bajas- de la fuerza enemiga que se enfrentaba al régimen, situación que remonta sus orígenes a 1973 en el accionar de la Triple AAA (Balvé y Balvé, 2005 y 2006; Marin, 2006; Rapoport, 2000; Sartelli, 2007; Sartelli, 2011) y que, a partir de la instauración del régimen militar en marzo de 1976, adquirirá la forma de la "desaparición de personas".

El régimen militar era consciente de la tarea que debía encarar. En las palabras de uno de los ministros de educación de la etapa, Llerena Amadeo:

“Está fuera de discusión que el pronunciamiento del 24 de marzo de 1976 puso fin a la crisis en que por entonces nos debatíamos, (...) al muy largo proceso de decadencia nacional en el cual los argentinos estábamos inmersos desde las últimas décadas." (Disertación y respuestas del Ministro de Cultura y Educación Dr. Juan Rafael Llerena Amadeo en el almuerzo mensual del Colegio de Abogados de la Ciudad de Buenos Aires [p. 4]. [1979, 2 agosto]).

La crisis y la decadencia eran identificadas con la emergencia de tendencias ideológicas "contrarias" a los intereses de la Nación, esto es, con el ascenso de "ideologías subversivas" que bregaban por una transformación social revolucionaria de la sociedad vigente. Los militares balanceaban que esos grupos se hallaban inclusive en la escuela. En ese sentido, hacia 1978, el régimen castrense difundió un documento denominado "Subversión en el ámbito educativo. Conozcamos a nuestro enemigo". En la práctica, constituía un manual para la detección de agentes peligrosos (subversivos) en la vida escolar. Se partía de un balance:

"La institución educativa ha sido alcanzada y afectada por la prédica y el accionar de nefastas tendencias ideológicas, (...) Como consecuencia (...) se observan omisiones y transgresiones a las normas que rigen el tratamiento y respeto que se debe a los símbolos nacionales." (Resolución no 1635, 1978 noviembre 3. (p. 2). Boletín de Comunicaciones, $n^{\circ} 61,1978,3$ de noviembre).

Por ello, en sintonía con esa caracterización, la educación cobró principalidad en la agenda de tareas generales encaradas por el personal militar. En esa dirección, el primer Ministro de Educación militar, Ricardo Pedro Bruera, sostuvo que la labor

2. El 29 de mayo de 1969 una huelga general convocada por los sindicatos de la provincia argentina de Córdoba derivaría en una insurrección de masas, conocida como el "Cordobazo". Durante aquella jornada, se establecería un combate abierto entre las fuerzas armadas y fracciones de la clase obrera aliadas con sectores estudiantiles. El "Cordobazo" inició una tendencia a la acción insurreccional de las masas, por fuera de los canales institucionales -partidos y sindicatosdando lugar al desarrollo de un proceso de características revolucionarias. Para ampliar, véase: Balvé, B. (et. al.) (2005). Lucha de calles, lucha de clases (Córdoba 1971-1969). Buenos Aires, Argentina: Ediciones RyR-CICSO. 
de la etapa era "reintegrar a la escuela argentina dentro del sentido nacional y de sus funciones al servicio del país" (Palabras pronunciadas por el Ministro de Cultura y Educación, Profesor Ricardo Bruera, al asumir su cargo (p. 4). 1976, marzo 30). La infiltración subversiva alejaba a la escuela de sus tareas tradicionales descarrilándola de su labor formativa. En consecuencia, se debía: "Iniciar el trazado de un nuevo modelo de organización del sistema educativo y este nuevo modelo (...) será (...) un proceso que iniciaremos en una tarea progresiva y paulatina." Los fines eran claros, los medios para realizar la tarea se diseñarían acompasadamente. Así, reprimir y reordenar constituían fines perentorios.

La impronta represiva del régimen militar sobre el sistema educativo ha sido advertida por numerosos trabajos desde diferentes perspectivas analíticas (Finocchio, S. y Lanza H. 1993; Invernizzi, H y Gociol, J. 2002; Invernizzi, H, 2005; Kaufmann, C. 1997; Kaufmann, C. y Doval, D. 2001; Pineau, P. y Mariño, M. 2006; Southwell M. (2007a; 2007b); Tedesco, J. C., Braslavsky, C. y Carciofi, R., 1983; Tiramonti, G. 2001). Algunos trabajos identifican, además, al período dictatorial como punto de inflexión en la historia educativa, trascendiendo la clave represiva (Pineau, P. 2006). Específicamente con la apertura y posterior consolidación de una tendencia neoliberal en materia educativa que se caracterizaría por la desaparición del Estado como agente educador principal. La brecha se cerraría en la década del noventa con un Estado mínimo (subsidiario), con la existencia de tendencias a la privatización creciente del servicio escolar y a la fractura y a la fragmentación de las trayectorias educativas. Así, se dejaría atrás en forma indeclinable la fase "normalista" caracterizada por la existencia de un Estado presente y principal, oficial educador-civilizador de su población (Puiggrós, A. 2003a; 2003b).

Consideramos que las distintas interpretaciones historiográficas pierden de vista las conexiones entre lo actuado en aquellos años "neoliberales" y lo acontecido en etapas previas y posteriores, es decir, la necesidad de realizar un análisis histórico de la educación que ligue "lo escolar" en relación a las tendencias más generales que afectan al conjunto de la sociedad. Nuestra mirada se nutre del materialismo histórico. En ese sentido, el modo material en que los hombres producen la vida condiciona el proceso social político y espiritual en general, así como las tareas que la escuela se da en se marco mayor. Tomando ese punto de partida, veamos qué ocurrió específicamente en los denominados "años de plomo".

\section{Regionalizar el CURrículum}

Tempranamente, la dictadura dio a conocer los lineamientos principales de su labor en materia educativa a través de un discurso público emitido por el Ministro, Pedro Bruera (Mensaje del Ministro de Cultura y Educación al país por la Red Nacional de Radios y Televisión, a las 21 hs. 1976 abril 13). Tres ejes resultaban medulares. Por un lado, pretendían reordenar la "realidad escolar" por ese momento "caótica" producto de la infiltración de ideologías "subversivas". En segundo lugar, instaurar un nuevo modelo de organización educativa. Finalmente, se mejorarían todos los procesos formativos.

Dicho plan emergía de un balance previo: la educación se encontraba en un "profundo estancamiento" y no acompañaba los requerimientos y las necesidades 
efectivas del país. De todos los niveles, la escuela secundaria requería una intervención urgente para convertirlo en un espacio de capacitación y de orientación de los jóvenes hacia el mundo del trabajo. Sin embargo, allí no se agotaban los problemas. En general, la estructura del sistema era altamente burocrática producto de la intervención excesiva del Estado central en detrimento de las provincias, más ligadas con el día a día escolar.

Las periódicas Asambleas de Ministros fueron el instrumento utilizado para la discusión y aprobación de las distintas medidas que se fueron implementando. Ya en mayo de 1976, el listado de prioridades a encarar en el nivel primario resultaba más que extenso. Dentro de la agenda de tareas, la revisión de objetivos y contenidos mínimos del nivel para armonizarlos con el tipo de hombre que el "Proceso de Reorganización Nacional" buscaba formar constituían núcleos medulares de la futura intervención. Así fue explicitado en la III Asamblea del Consejo Federal de Educación (III Asamblea Extraordinaria del Consejo Federal de Educación, Buenos Aires (1976, mayo 10)) realizada en la Ciudad de Buenos Aires. En esa reunión, la Comisión de Contenidos fijó la necesidad de revisarlos para que respondieran, además, a un "programa regionalizado".

Apenas unos meses más tarde, en el marco de la V Asamblea Ordinaria del Consejo Federal de Educación, realizada en San Miguel de Tucumán (Informe final y Anexos de la $V$ Asamblea Ordinaria del Consejo Federal de Educación (1976, septiembre 15 al 17). Ministerio de Cultura y Educación de la Nación), se debatió acerca de cuáles debían ser los fines, objetivos y agentes del sistema educativo. Esa delimitación constituía "un factor fundamental en la erradicación de las causas de la subversión". Ahora bien, quiénes eran considerados agentes educativos. En primer término, la familia como agente educador natural y primario. Pero, ese reconocimiento no implicaba para el Estado una función subsidiaria; éste tenía el deber y el derecho de formar integralmente a sus ciudadanos. Junto a él, se ubicaban la Iglesia Católica, otras confesiones religiosas y las asociaciones con personería jurídica o de otro tipo idóneas para el desarrollo de esa tarea. Del primero al último promoverían y facilitarían la participación activa y estructurada de la familia y de la comunidad.

La Asamblea dispuso, además, la coordinación, transferencia y regionalización de los servicios educativos (Resolución $n^{-}$3). Transcurrieron dos años hasta que se resolvió la transferencia (Decreto-Ley $\mathrm{n}^{\circ} 21.809$ y 21.810, Anales de Legislación Nacional, Tomo XXXVIII-B, p. 1.454-1.457 (1978, junio)). Retomaremos este punto en un acápite posterior. En materia de "reconversión curricular", la Resolución no 4 convocó a una Comisión Permanente de Contenidos Mínimos para que se expidiera, en treinta días, con un despacho a tales efectos.

En diciembre, una nueva Asamblea determinó fines y objetivos del sistema educativo y "contenidos mínimos" para la escuela primaria "reorganizada" (Objetivos pedagógicos del nivel primario y del nivel medio. Contenidos mínimos del nivel primario común (Resolución no 284/77-Nueva Serie Divulgación). (1977, diciembre 16 y 17) Ministerio de Cultura y Educación-Consejo Federal de Educación: IV Asamblea Extraordinaria. Informe final. Anexos (1977, 16 y 17 de diciembre)). Como pauta general, las reformas intentaban que las provincias tuvieran una mayor intervención en la innovación curricular sobre los contenidos impartidos en sus escuelas. En relación a ese punto, se especificaba que cada jurisdicción debía realizar "adecuacio- 
nes indispensables para cada Estado". Las "adecuaciones" no eran otra cosa que el poder jurisdiccional de las provincias ejercido sobre el currículum en las múltiples respuestas qué éstas podían dar.

Además, se apostaba a que la mayor participación de las provincias alcanzara la capacitación laboral y la orientación vocacional brindada por cada escuela. El documento expresaba:

"La escuela para que el alumno consiga un desarrollo motriz pleno debe seleccionar y organizar actividades que permitan el control del cuerpo y la manipulación segura de los objetos. En tal sentido, se han de orientar las actividades prácticas que desarrollen habilidades y destrezas útiles para una elemental capacitación laboral según necesidades regionales" (p. 9).

Tal como puede verse, la escuela no brindaría meramente una formación enciclopedista. Tampoco la educación motriz se limitaba a asignaturas como educación física. La formación integral contemplaría capacidades para desempeñarse en el mundo del trabajo. Ahora bien, ella debía realizarse en estrecha interconexión con el mundo local en el que el joven se insertaría.

En suma, la Asamblea se encargó de elevar la serie de contenidos mínimos que regirían en todo el país a partir de 1977. Por cuestiones de espacio, no analizaremos aquí el detalle curricular materia por materia. Lo que sí destacamos es que detrás de esa aparente unificación regía una tendencia más bien contraria. Desde la década del sesenta, en el país, se bregaba por generar una mayor adecuación de los contenidos curriculares a las realidades de cada una de las provincias y/o regiones. A partir de ese momento, las intervenciones curriculares desplegadas por la Nación se encaminaban a fijar contenidos "mínimos, básicos y comunes", tal como ocurrió, previamente, en 1959-1961, 1964, 1969-70, 1972 y 1975³. La intención detrás de esos esbozos era diseñar un currículum lo suficientemente flexible para que la escuela sirviera mejor a las necesidades de las "fuerzas vivas" o productivas. Desarrollaremos este punto hacia el final.

\section{VigILANCIA DE CUMPLIMIENTO EFECTIVO}

El régimen castrense se encargó de vigilar el cumplimiento de las medidas que iba aprobando. Creó un programa de asistencia técnica para conocer el nivel de avance en la adaptación regional de los contenidos mínimos y básicos (Programa Regional de desarrollo educativo. Proyecto multinacional de investigación educativa-Jornadas de Evaluación de Innovaciones Curriculares. (1978, abril 10 al 12). Ministerio de Cultura y Educación, Secretaría de Estado de Educación, Subsecretaría de Educación, Organización de los Estados Americanos-Secretaría General-Departamento de Asuntos Educativos, Dirección Nacional de Investigación, experimentación y perfeccionamiento educativo (Buenos Aires)). En un primer informe se recabaron datos suministrados por seis provincias argentinas: Buenos Aires, Catamarca, Corrientes,

3. Para una consulta detallada de los cambios y continuidades remitimos a la consulta de la tesis doctoral de la autora defendida en la Universidad de Buenos Aires. 
Misiones, Santa Fe y San Juan. Intentaban determinar si se lograba incidir en el quehacer educativo. También, el grado de "disponibilidad al cambio" de sus protagonistas y la capacidad de conducción de sus responsables.

El primer informe del que tenemos registro, elaborado por el Consejo Federal de Educación data de 1980 (Evaluación de los contenidos mínimos de nivel primario. (Nueva Serie. Estudios y Documentos no 1). (1980, Buenos Aires) Ministerio de Cultura y Educación-Dirección Nacional de Investigación, Experimentación y Perfeccionamiento Educativo). Habían transcurrido casi cuatro años desde las primeras medidas. Y, sin embargo, la situación aún no resultaba conclusiva. Se caracterizaba que el área curricular más avanzada en materia de reforma era la de ciencias sociales. Sólo tres provincias no habían concluido la reconversión: Santa Fe, Neuquén y los establecimientos dependientes del Consejo Nacional de Educación. No resulta extraño suponer que dicha asignatura se ubicó a la vanguardia debido a la misión que el régimen le había proporcionado: reforzar los valores tradiciones, la nacionalidad, la democracia como estilo de vida y el sentimiento de pertenencia. Ahora bien, ¿cuáles eran los cambios introducidos en el currículum? Mientras veinticuatro provincias mencionaban modificaciones en el desarrollo temático, veintitrés destacaban nuevas actividades sugeridas, veinte señalaban cambios en las orientaciones metodológicas y quince en las orientaciones bibliográficas y técnicas de evaluación. Algunas provincias agregaban aspectos tales como: guías de análisis de los programas curriculares (Buenos Aires), fundamentos filosóficos, psicológicos, pedagógicos y culturales (Corrientes, Chaco y Río Negro) y la planificación de la unidad escolar (Corrientes). La provincia de Chaco, por su parte, se había encargado de pautar, específicamente, los fines y objetivos de la educación provincial. Los cambios en la provincia de La Rioja abundaban sobre la organización escolar, la orientación laboral y los problemas de aprendizaje. Chubut, por su parte, destacaba la regionalización, la agrupación de grados y la integración del área de expresión.

En general, coincidían en que los lineamientos se aplicaban en todas las escuelas provinciales o, en su defecto, en la gran mayoría. En lo que refería a las ventajas, en el plano didáctico destacaban: la uniformidad en la nomenclatura de las áreas y asignaturas curriculares, la correlación interdisciplinaria, su estructuración lógica así como la utilización de métodos científicos para la formación de los niños y la realización de adaptaciones acorde a las necesidades y posibilidades del núcleo escolar. La mayor flexibilidad aparecía como uno de los valores de la experiencia.

Así, la larga marcha de la regionalización comenzó a convertirse en realidad en el sistema educativo durante la última dictadura militar. Realidad que, desde 1959, se apuntalaba cuando se enfatizaba sobre la necesidad de sancionar lineamientos "mínimos". En ese oportunidad, se destacaba:

“La comisión prefirió preparar un programa básico uniforme (...) insistiendo en la necesidad de una adaptación local del mismo, constante y dinámica, comenzando el estudio de las diversas asignaturas por lo que el medio inmediato ofrece a la observación y el interés del niño y preconizando los ejercicios, problemas y prácticas manuales adecuados a las costumbres, necesidades y productos de la localidad" (Programa de Educación Primaria. Anteproyecto, (1959) Consejo Nacional de Educación. Buenos Aires: Argentina, p. 11). 
La elaboración de diagnósticos por parte de la Nación sobre la marcha de la regionalización continuó (Reunión de trabajo para el análisis de los aspectos técnicos de los contenidos mínimos del nivel primario y del ciclo básico del nivel medio. Dirección Nacional de Investigación, Experimentación y perfeccionamiento educativo-Sector Currículum. (1981, junio 8 al 12)). Pero, las provincias reclamaban la sanción de un marco normativo más general sobre el cual fundar las adaptaciones. Y, en 1981, se comenzó a dar forma a un proyecto de ley educativa: Ia Ley Federal. En realidad, ya hacia fines del '80, la III Asamblea Ordinaria del Consejo Federal de Educación había elevado al Poder Ejecutivo algunas de las pautas que debían tenerse en cuenta para la elaboración de una futura ley reafirmando la necesidad de regionalizar el sistema y de dividir tareas. El Estado nacional fijaría las bases de cada plan determinando objetivos y contenidos mínimos. Cada jurisdicción aprobaría, a su vez, sus propias bases desarrollando, adecuando y ampliando las nacionales. Por último, cada uno de los establecimientos haría, a su vez, su propia adaptación.

Como vemos, entonces, todas las medidas adoptadas se encaminaban a garantizar una injerencia cada vez mayor de las distintas realidades locales sobre el currículum.

\section{LA LARGA MARCHA DE LA DESCENTRALIZACIÓN}

La regionalización curricular lograda por la dictadura puso fin a una larga serie de ensayos o de cambios proyectados previamente en esa dirección. Pero, cabe preguntarse por qué el intento logró cuajar en este momento y no en los anteriores.

Resulta importante destacar que la regionalización del currículum se solapó con otro proceso de gran envergadura, y también de larga data, que le dio sentido: la descentralización del sistema escolar. Desde, por lo menos 1956, se produjeron numerosos intentos de transferencia de la gestión y administración de los establecimientos educativos gobernados por el Estado central a la égida de las provincias, revirtiéndose así la estructura centralizada con la que históricamente se había configurado el sistema educativo argentino.

En efecto, si bien la Constitución Nacional de dicho país, en su artículo 5ㅜㅡ, ya en 1853 estableció que la educación básica se encontraba a cargo de las provincias, la estructuración del mismo corrió a cargo de la autoridad nacional a partir de la sanción de la Ley Lainez, en 1905 (Ley no 4.878 promulgada en octubre de ese año, implementada en febrero del año siguiente). Las provincias argüían falta de recursos económicos para garantizar una cobertura total del sistema educativo a lo largo y ancho del territorio bajo su administración. Entonces, la nueva Ley habilitó a la Nación a "socorrer" a las provincias que reclamaran auxilio de la Nación a los efectos de garantizar el cumplimiento de las metas educativas fijadas en la Ley 1.420 de educación común, básica y obligatoria promulgada el 8 de julio de 1884. El puntal legal permitió que, en medio siglo, la masa de la población se incorporara al sistema educativo consiguiendo elevados índices de alfabetismo; situación que distinguió a Argentina de la trayectoria seguida por otros países latinoamericanos. 
Pero, el año 1956 constituyó el primer intento por revertir dicha tendencia. Uno de los primeros puntos de inflexión se inició junto a la autoproclamada "Revolución Libertadora"4 cuando se restauró el Consejo Nacional de Educación, a través de la Ley $\mathrm{n}^{\mathrm{0}}$ 7.977. Al antiguo organismo se le otorgó, ahora, la potestad de transferir escuelas nacionales a las provincias. Argumentaban que la "dictadura" peronista, en su afán de absorción de la dirección de los organismos estatales, había avasallado todos aquellos "cuya estructura descentralizada, federativa y autárquica, admitía una mayor participación ciudadana en el manejo de la cosa pública" (Anales de Legislación Nacional. (Tomo XIV, pp. 351-352, (1956, Septiembre)).

El ojo del régimen militar focalizó sobre aquellas medidas que reforzaron la estructura centralizada. Durante la primera y segunda presidencia de Juan Domingo Perón, entre 1946 y 1955, se produjeron una serie de reformas dirigidas a aumentar el centralismo. Uno de los hitos más salientes fue la creación del Ministerio Nacional de Educación, en el año 1949 (Anales de Legislación Nacional. (Tomo IX-A, p. 196) (1949)) separándolo del Ministerio de Justicia como una entidad independiente. Se suprimieron, también, todos los Consejos Escolares Provinciales, reforzando así el comando centralizado.

En 1954, ya durante la segunda presidencia de Perón, se tomaron otras medidas. En primer término, se reestructuró el Ministerio de Educación y se reformaron algunos de los organismos que lo estructuraban (Anales de Legislación Nacional. (Tomo XIV-A, p. 554) (Año 1954)). Allí también se dispuso que "la acción educativa nacional debe ser desarrollada con unidad de concepción en todo el país, sin que ello importe desatender las necesidades regionales o locales correspondientes".

Por el contrario, la Ley 7.977, de 1956, sostuvo que la política educacional debía ajustarse a los principios federativos. Y, si bien Nación y provincia tenían acciones concurrentes en materia educativa, "[resultaba] preciso que aquella se oriente y organice estimulando la acción provincial y teniendo en cuenta, con respecto a las escuelas que crea y dirige, las tradiciones y modalidades regionales". Solo así se posibilitaría la injerencia de la familia y de las comisiones vecinales en el gobierno de la educación. Lógicamente, se fijó como una de las atribuciones del Consejo "[el] tener facultades (...) para convenir, según ciertas reglas, la transferencia de sus escuelas a las provincias". En este sentido, el artículo 11ํe establecía que se "podrá transferir a las provincias todas o algunas de las escuelas (...) ad referendum del poder ejecutivo".

Si bien en esos años no se transfirieron escuelas, los primeros traspasos no demoraron demasiado. Bajo la presidencia del radical intransigente Arturo Frondizi (quién accedió al "sillón de Rivadavia" entre mayo de 1958 y marzo de 1962), la Ley 4.874 de 1961 inició el proceso firmándose distintos convenios particula-

4. El golpe de Estado se produjo el 16 de septiembre de 1955. Encabezado por Eduardo Leonardi y Pedro Eugenio Aramburu, implicó la proscripción política de las masas que se identificaban con el peronismo, hecho que se expresó en la prohibición hacia el Partido Peronista de participar en la vida política del país hasta 1973. 
res con las respectivas provincias a los efectos de implementar la mentada transferencia. El convenio se establecía entre el Consejo Nacional de Educación y el Ministro de Educación de la respectiva provincia a la cual se le transfería el servicio. El acuerdo celebrado debía ser ratificado por las legislaturas provinciales. También debían hacerlo los Ministros de Economía e Interior (Anales de Legislación Nacional (Año 1961), pp. 808-809). En el año 1962, el Decreto № 495 del 17 de enero, traspasó compulsivamente los establecimientos primarios a las provincias, hubieran o no firmado convenios particulares. En el artículo $5^{\circ}$ se detallaba que "el personal que no acepte el cambio de jurisdicción será dado de baja e indemnizado en las condiciones establecidas en el Art. 49 de la Ley 16.432". El 7 de agosto del mismo año se dejó sin efecto el proceso de transferencias una vez que Frondizi fuera derrocado de la presidencia por el golpe militar que catapultó a la presidencia a José María Guido. El Decreto de derogación recibió el № 7.814. La justificación de la contramarcha fue puramente técnica: se adujo que los convenios de transferencia no habían sido debidamente ratificados por las legislaturas provinciales.

Sin embargo, ese no fue el fin de ese tipo de políticas. La siguiente avanzada "descentralizadora" corrió a cargo del presidente de facto Juan Carlos Onganía quien se encontraba a la cabeza de la "Revolución Argentina" en el poder desde junio de 1966. En esa oportunidad, la Ley 17.878 del 5 de septiembre de 1968 traspasó establecimientos nacionales a las provincias.

Los mecanismos formales dispuestos fueron exactamente los mismos que los implementados años atrás. En esta oportunidad, se especificó que era un "propósito definido lograr la centralización en la conducción y la descentralización en la ejecución" (Anales de Legislación Argentina. (Volumen XXVIII-C, pp. 3290-3291) (Año 1968)). Como en el caso anterior, las reformas educativas confluyeron con un proceso de racionalización administrativa mayor (Ley no 18.586) (Anales de Legislación Argentina. (Año 1970) (Volumen XXX-A, pp. 148-149)).

Como vimos a lo largo del presente artículo, la descentralización también fue un principio defendido durante la dictadura de 1976. La transferencia de establecimientos se inició mediante las Leyes 21.809 y 21.810 del 5 de Junio de 1978 (Anales de Legislación Argentina. (Año 1978, Volumen XXXVIII-B) (pp. 1.454-1.544)) y a través del Decreto 1.230 del mismo año. La historiografía argentina ha supuesto que ese proceso dio lugar a un retiro del Estado en el manejo de la cosa pública y a niveles crecientes de privatización educativa. En otro lugar nos hemos ocupado de discutir tal idea verificando un reposicionamiento estatal ${ }^{5}$. En relación al problema que aquí hemos estudiado, el proceso descripto en el plano legal tuvo su correlato en la cotidianeidad escolar. Los datos estadísticos sobre la evolución de la matrícula y de las escuelas en cada una de las autoridades educativas (Nación, provincias y circuito privado) permiten afirmar que la descentralización logró imponerse sobre el sistema educativo.

5. Obviamos cita para conservar anonimato. 


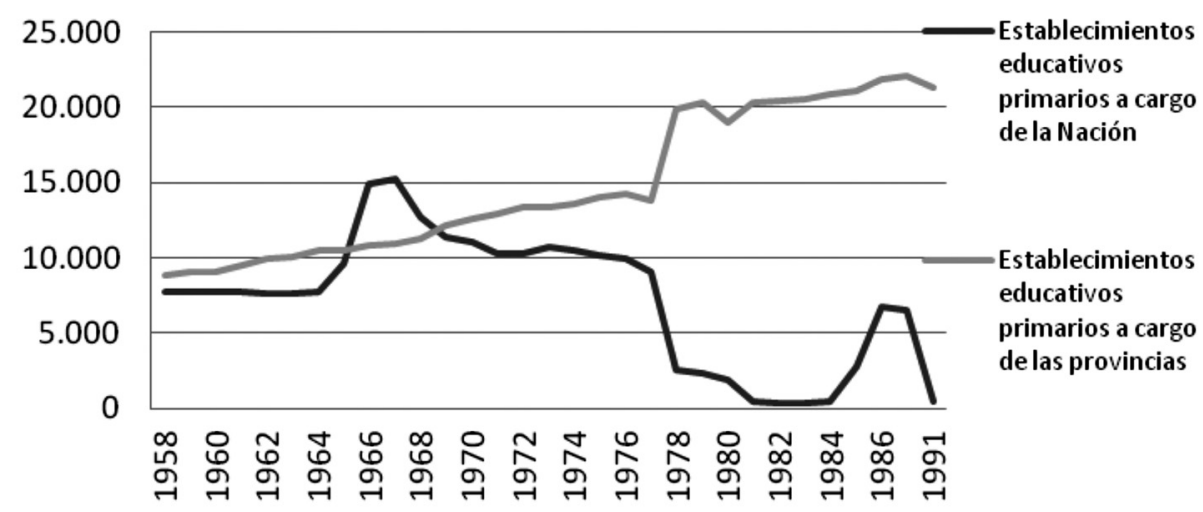

Gráfico 1. Argentina. Evolución de establecimientos por autoridad. Nivel Primario. Años: 1958-1991. Fuente: Elaboración propia en base a fuente citada
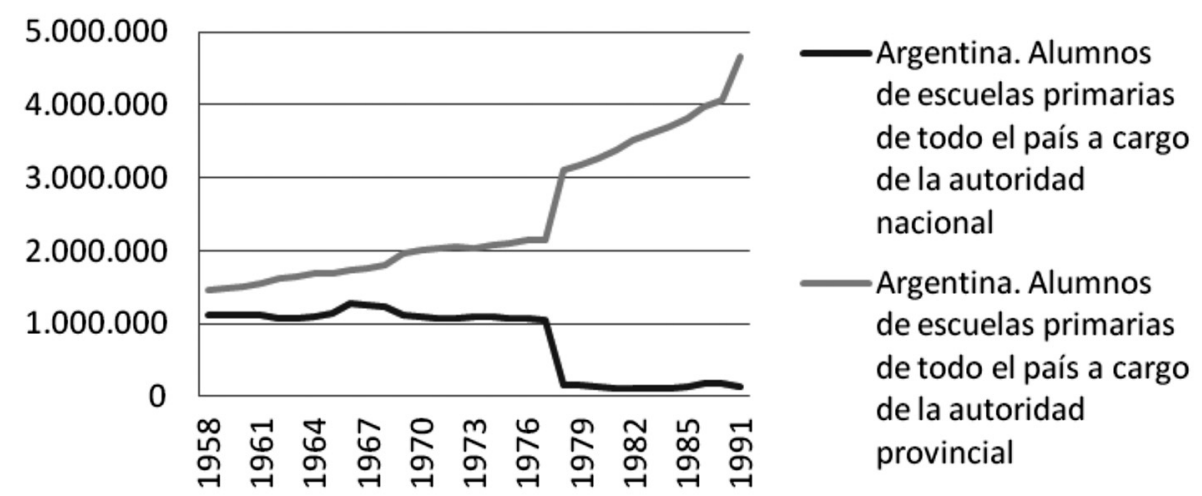

Gráfico 2. Argentina. Alumnos de educación primaria de todo el país por tipo de autoridad (nacional o provincial). Años: 1958-1991. Fuente: Elaboración propia en base a fuente citada

Resulta importante destacar que, el período 1976-1983, aparece como un momento clave. Tal como ponen de manifiesto los gráficos $\mathrm{n}^{\mathrm{o}} 1$ y 2, la última dictadura militar se correspondió con una completa descentralización en la administración educativa del nivel primario. El gráfico $\mathrm{n}^{\mathrm{o}} 1$ pone de relieve que la amplia mayoría de los establecimientos educativos del nivel primario recayeron bajo la administración provincial una vez efectuados los traspasos legales. Una evolución similar afectó a la matrícula del nivel primario tal como da cuenta el gráfico no 2 . Resulta importante destacar, además, que los datos estadísticos dan cuenta de que la tendencia creciente a la descentralización del sistema no se revirtió en los momentos en los que se desafectó el proceso en el plano legal tales como 1962 y 1970. La administración creciente de las provincias en la gestión educativa se impuso como una tendencia ascendente que atravesó activamente al personal político 
que la impulsó así como a aquel que se manifestó en forma opuesta o pasiva frente a dichas iniciativas.

No extraña, entonces, que el momento de mayor descentralización del sistema coincidiera con la introducción de estrategias de regionalización a nivel curricular. Ambos aspectos permiten reconstruir la introducción de una estrategia global sobre el sistema educativo que afectó el plano administrativo y al curricular o cognitivo en un todo y que tienen un punto de Ilegada hacia 1976.

\section{A MODO DE CONCLUSIONES PROVISORIAS: DESCENTRALIZACIÓN Y REGIONALIZACIÓN COMO PARTE DE UNA MISMA ESTRATEGIA}

En el presente artículo nos hemos ocupado de recomponer los principales cambios de orientación que la última dictadura militar le propinó al currículum de la escuela primaria. Como dijimos, el proceso de reorganización social fue acompañado de una reorganización curricular. En un sentido más amplio, en la etapa bajo estudio, se le otorgó un rol central a la educación. Ella era vista como un reducto atacado por la subversión plausible, entonces, de ser depurado y colocado al servicio de la restauración del orden. De la gran cantidad de intervenciones encaradas en esos años, aquí nos ocupamos de un aspecto poco explorado: la regionalización del currículum. Hemos reconstruido cómo el régimen castrense buscó que las provincias tuvieran cada vez mayor injerencia en el diseño de los contenidos curriculares a enseñarse en sus territorios. Se argumentó que satisfacer las necesidades regionales tornaría significativa a la educación. Por ello, durante los "años de plomo" la dictadura promulgó unos nuevos "contenidos mínimos y comunes" para todo el país con el objeto de que ellos fueran, luego, adaptados en las distintos espacios locales.

Como mostramos aquí, la dictadura militar se encargó de monitorear que las provincias intervinieran sobre los contenidos. Creó organismos de seguimiento y control de las experiencias que puso en marcha. Pero la nueva orientación curricular (flexibilidad, regionalización y adaptabilidad) no resultaba novedosa. Si bien no nos hemos ocupado de ello, desde fines de la década del cincuenta se intentó imponer esa tendencia. Resulta importante enfatizar que la "obsesión oficial" por que las provincias intervinieran en la fijación del currículum educativo se expresó en un momento en el que la gestión y administración de las escuelas primarias recayó en forma casi completa bajo la órbita provincial. Es por ello que ambos procesos deben ser examinados en forma conjunta.

A nuestro entender, descentralización y regionalización no conforman productos originales de la última dictadura militar. Por el contrario, trabajo anterior, nos permitió ver que las tareas encaradas entre 1976 y 1983 fueron largamente añoradas y ensayadas por diverso personal político. La dictadura constituyó más bien un momento de síntesis. Si una y otra orientación lograron imponerse en esos años fue resultado de la correlación de fuerzas alcanzada en la etapa. Como dijimos al inicio del trabajo, la última dictadura militar tenía por misión cerrar la brecha abierta, en 1955, como crisis hegemónica y de acumulación de capital. La eliminación de la alianza social que se le imponía al régimen era una tarea perentoria. Por ello, buscó depurar su intervención en el ámbito educativo. Pero también le resultaba apre- 
miante la eliminación de capital sobrante y la creación de una "población sobrante" de magnitudes hasta ese momento nunca antes vistas en la sociedad argentina. Las tareas en la "esfera económica" impactaron sobre la relación que la escuela trazaba con el sistema productivo y con el resto de la sociedad. Si hasta la década del cincuenta la relativa homogeneidad de la clase obrera imponía a la escuela la misión de garantizar una educación homogénea para el conjunto de la población, su creciente fractura cuestionó tal legado. Se procedió a revisar la idea de una formación universal-genérica y se dio lugar a una formación parcializada. Años antes, durante la presidencia de Onganía se había intentado avanzar en una reforma de características similares que alteraba también la estructura tradicional del sistema educativo. Sin embargo, la lucha encarada por los docentes puso serios límites a la experiencia hacia 1971. La dictadura del 76 apoyó su éxito en materia educativa sobre el nuevo esquema de fuerzas alcanzado.

Debemos enfatizar que la descentralización-regionalización no implicó una desaparición del Estado sino una reconfiguración de su función. El Estado mutó de estrategia: del comando centralizado al de las distintas localidades. Este último punto no resulta menor. La década del ochenta se encontró atravesada por la elaboración de estudios que reseñaron las bondades e inconvenientes que el proceso había dejado. Síntoma del éxito en la imposición del programa previo.

\section{REFERENCIAS BibLIOGRÁFICAS}

Anales de Legislación Argentina (1968), Vol. XXVIII-C, pp. 3.290-3.291.

Anales de Legislación Argentina (1970), Vol. XXX-A, pp. 148-149.

Anales de Legislación Argentina (1978), Vol. XXXVIII-B.

Anales de Legislación Nacional (1949), Tomo IX-A, p. 196.

Anales de Legislación Nacional (1954), Tomo XIV-A, p. 554.

Anales de Legislación Nacional (1956), Tomo XIV, pp. 351-352.

ARGENTINA. CONSEJO FEDERAL DE CULTURA Y EDUCACIÓN (1976). Asamblea Ordinaria [5ª 1976]: Informe final y Anexos.

ARGENTINA. MINISTERIO DE CULTURA Y EDUCACIÓN. CONSEJO FEDERAL DE CULTURA Y EDUCACIÓN (1976). III Asamblea Extraordinaria. Buenos Aires: Ministerio de Cultura y Educación Argentina.

ARGENTINA. MINISTERIO DE CULTURA Y EDUCACIÓN (1980). Evaluación de la aplicación de los contenidos mínimos de nivel primario. Buenos Aires: Dirección Nacional de Investigación, Experimentación y Perfeccionamiento Educativo, Centro Nacional de Documentación e Información Educativa. (Nueva Serie Estudios y Documentos, no 1). Disponible en: http://www.bnm.me.gov.ar/giga1/documentos/ EL002178.pdf.

ARGENTINA. MINISTERIO DE CULTURA Y EDUCACIÓN. (1977). Objetivos pedagógicos del nivel primario y del nivel medio: contenidos mínimos del nivel primario común : Resolución no 284/77. En IV Asamblea Extraordinaria. Informe final. Anexos, 1977, 16 y 17 de diciembre. Buenos Aires: Centro Nacional de Documentación e Información Educativa. (Nueva Serie Divulgación). 
ARGENTINA. MINISTERIO DE CULTURA Y EDUCACIÓN (1959). Programa de Educación Primaria. Anteproyecto.

ARGENTINA. MINISTERIO DE CULTURA Y EDUCACIÓN y ORGANIZACIÓN DE LOS ESTADOS AMERICANOS (1978). Programa Regional de desarrollo educativo. Proyecto multinacional de investigación educativa. Jornadas de Evaluación de Innovaciones Curriculares, (1978, abril 10 al 12). Buenos Aires: Dirección Nacional de Investigación, Experimentación y Perfeccionamiento Educativo.

BALVÉ, B. (et al.) (2005). Lucha de calles, lucha de clases (Córdoba 1971-1969). Buenos Aires: RYR-CICSO.

BALVÉ, B. (et al.) (2006). El 69. Huelga política de masas: rosariazo-cordobazo-rosariazo. Buenos Aires: RYR-CICSO.

BORTHWICK DE MALTONI, M. (1995). Legislación educativa Argentina. Buenos Aires: Braga Ediciones.

BRAVO, H. F. (1994). La descentralización educacional. Sobre la transferencia de establecimientos. Buenos. Aires: CEAL.

BRUERA, R. P. (1976). Mensaje del Ministro de Cultura y Educación al país [transmitido por la Red Nacional de Radios y Televisión el 13-04-76]. Buenos Aires: Ministerio de Cultura y Educación Argentina. Disponible en: http://www.bnm. me.gov.ar/giga1/documentos/EL000065.pdf.

BRUERA, R. P. (1976). Palabras pronunciadas por el Ministro de Cultura y Educación al asumir su cargo. Buenos Aires: Ministerio de Cultura y Educación Argentina. Disponible en: http://www.bnm.me.gov.ar/giga1/documentos/EL000021.pdf.

DE LUCA, R. (2008). Brutos y baratos. Descentralización y privatización en la educación argentina (1955-2001). Buenos Aires: RyR.

DECRETO-LEY no 21.809 y 21.810 (junio 1978). Anales de Legislación Nacional, Tomo XXXVIII-B, pp. 1454-1457.

FELDFEBER M. e IVANIER A. (2003). "La descentralización educativa en Argentina: el proceso de transferencia de las instituciones de formación docente" en Revista Mexicana de Investigación Educativa, (Vol. 8, no 18). Disponible en: www.redalyc.com.

FINOCCHIO, S. y LANZA H. (1993). La enseñanza de la historia en la Argentina de hoy. Buenos Aires: Miño y Dávila.

INVERNIZZI, H. y GOCIOL, J. (2002). Un golpe a los libros. Buenos Aires: Eudeba. INVERNIZZI, H. y GOCIOL, J. (2005). Los libros son tuyos. Buenos Aires: Eudeba.

KAUFMANN, C. (1997). Una pedagogía de la renuncia. El perennialismo en Argentina (1976-1983). Rosario: Laborde Editor.

KAUFMANN, C. y DOVAL D. (2001). Dictadura y educación. Buenos Aires: Miño y Dávila.

LLERENA AMADEO, J. R. (1979). El diálogo y los problemas educativos. Disertación y respuestas del señor Ministro de Cultura y Educación Dr. Juan Rafael Llerena Amadeo en el almuerzo mensual del Colegio de Abogados de la ciudad de Buenos Aires el 2 de agosto de 1979. En Discursos ministeriales. Buenos Aires: Ministerio de Cultura y Educación Argentina. Disponible en: http://www.bnm.me.gov.ar/ giga1/documentos/EL000027.pdf. 
MARÍN, J. C. (1988). Los hechos armados: un ejercicio posible. Buenos Aires: La Rosa Blindada/P.I.CA.SO.

PAVIGLIANITTI, N.; TIRAMONTI, G. y TEDESCO, J. C. (1988). Diagnóstico de la administración central de la educación. Buenos Aires: Ministerio de Educación y Justicia.

PUIGGRÓS, A. (2003a). Dictaduras y utopías en la historia reciente de la educación argentina (1955-1983). Buenos Aires: Galerna. (Colección Historia de la Educación en la Argentina, VIII).

PUIGGRÓS, A. (2003b). ¿Qué pasó en la educación argentina? Breve historia desde la conquista hasta el presente. Buenos Aires: Galerna.

PINEAU, P. y MARIÑO, M. (2006). El principio del fin. Políticas y memorias de la educación en la última dictadura militar (1976-1983). Buenos Aires: Colihue.

RAPOPORT, M. (2000). Historia económica, política y social de la Argentina: (18802000). Buenos Aires: Macchi.

RESOLUCIÓN no 1635 (3 noviembre 1978). Boletín de Comunicaciones, 61.

REUNIÓN de trabajo para el análisis de los aspectos técnicos de los contenidos mínimos del nivel primario y del ciclo básico del nivel medio (1981). Buenos Aires: Dirección Nacional de Investigación, Experimentación y Perfeccionamiento Educativo, Sector Currículum.

RODRÍGUEZ, L. G. (2008). La escuela intermedia revisitada: racionalización y revisión curricular en la provincia de Buenos Aires durante la última dictadura militar. Trabajos y comunicaciones, 34, 35-61. Disponible en: http://www.fuentesmemoria. fahce.unlp.edu.ar/art_revistas/pr.3723/pr.3723.pdf [23-05, 2012].

SALONIA, A. (1996). Descentralización educativa, participación y democracia: escuela autónoma y ciudadanía responsable. Buenos Aires: Academia Nacional de Educación.

SARTELLI, E. (2007). La Plaza es nuestra. Buenos Aires: RyR.

SARTELLI, E. (2011). La crisis orgánica de la sociedad argentina. Buenos Aires: Editorial de la Facultad de Filosofía y Letras, Universidad de Buenos Aires.

SOUTHWELL, M. (2007a). Postdictadura y política educativa: una relocalización de viejos de imaginarios en pugna. Revista del Instituto de Políticas Públicas, 1 (1), 54-71.

SOUTHWELL, M. (2007b). "Con la democracia se come, se cura y se educa..." Disputas en torno a la transición y las posibilidades de una educación democrática. En La Argentina democrática: los años y los libros. Buenos Aires: Prometeo.

TEDESCO, J. C.; BRASLAVSKY, C. y CARCIOFI, R. (1983). El proyecto educativo autoritario. Argentina, 1976-1982. Buenos Aires: Facultad Latinoamericana de Ciencias Sociales.

TIRAMONTI, G. (2001). Modernización educativa de los noventa. ¿El fin de la ilusión emancipadora? Buenos Aires: FLACSO. 